\title{
Bases para la relación investigación - postgrado
}

Bases for the relationship between research and graduate education

Román Antonio Hernández-Dávila, Adriana del Pilar Cerinza-Contento.

a Doctoren Pedagogia, rahd17@gmail.com, orcid.org/0000-0002-2248-5841, Universidad Francisco de Paula Santander, Cúcuta, Colombia.

${ }^{a}$ Doctor en Educación, adri.cerinza@mail.udes.edu.co, orcid.org/0000-0002-9630-5655, Universidad de Santander, Cúcuta, Colombia.

Cómo citar: R.A. Hernández-Dávila y A.P. Cerinza-Contento, "Bases para la relación investigación - postgrado", Respuestas, vol. 23, no. S1, pp. 97-104, 2018. https://doi.org/10.22463/0122820X.1524

Recibido: Febrero 20, 2018; Aprobado: Julio 28, 2018.

\section{RESUMEN}

Palabras Clave:

Instituciones de educación superior

Investigación

Postgrado

Redes Académicas

Antecedentes: la investigación e innovación, son ejes de desarrollo socioeconómico del país. Colombia en las últimas décadas goza de cierto avance en $\mathrm{I}+\mathrm{D}$, aunque se requiere en la educación superior una masa crítica que asuma el desafío de la sociedad del conocimiento para enriquecer la relación transformadora entre academia-Estado-desarrollo integral-sociedad. Este artículo presenta una reflexión sobre los conceptos investigación-postgrado (CRES.UNESCO 2008, CESU 2014, COLCIENCIAS 2015), en la intención de lograr el Objetivo de (i) identificar las bases para una relación indisoluble entre ambos, con resultados contundentes en innovaciones, hallazgos científicos e impactos positivos en la calidad de vida y desarrollo sostenible (ii), que agreguen valor al intercambio científico y el diálogo de saberes; y (iii) formule recomendaciones sostenidas en alianzas estratégicas, para crear postgrados pertinentes, redes telemáticas respecto de objetos de estudios compartidos, comunidades de trabajo colaborativo inter y transdisciplinario, crear fuentes múltiples de financiamiento y ampliar grupos, líneas y proyectos de investigación. Desarrollo: el corpus del artículo presenta a las instituciones educativas de educación superior como las principales vitrinas propicias para proponer y mostrar proyectos factibles en investigación, innovación y desarrollo. Conclusiones: la cooperación indisoluble entre postgrados e investigación con criterios de pertinencia, juegan un papel importante para afrontar con propiedad los desafíos establecidos por la sociedad globalizada, en donde las ventajas comparativas entre empresas y países, se encuentran en gestionar con eficiencia el conocimiento que es el capital intangible, motor del valor agregado, movilizador de las economías y del desarrollo sustentable.

\section{ABSTRACT}

\section{Keywords:}

Higher education institutions Research Graduate degrees

Academic Networks
Background: Research and innovation are pillars of the socio-economic development of the country. During the last few decades, Colombia has enjoyed advancements in $\mathrm{R}+\mathrm{D}$, but higher education needs to develop critical mass in order to meet the challenge of a knowledge-based society and be able to enrich the transformational relationship among academia-State-integral development-society. This essay presents an insight into the concepts of research-graduate education (CRES.UNESCO 2008, CESU 2014, COLCIENCIAS 2015), with the aim of achieving the Objectives of (i) identifying the basis for an interwoven relationship between the two, with convincing results in innovations, scientific achievements and positive impact on the quality of life and sustainable development (ii) adding value to scientific collaboration and knowledge sharing; and (iii) formulating sustainable recommendations to strategic alliances, in order to develop relevant graduate programs, subject-based networks of shared studies, collaborative communities among and across disciplines, multiple sources of financing and to increase groups, lines and projects for research. Development: the core of the essay proposes to have the institutions of higher education as the main suitable window to propose and showcase feasible projects in research, innovations and development. Conclusions: The interwoven cooperation between graduate programs and research with pertinent criteria play an important role in facing the challenges established by an increasingly globalized society, where comparative advantages between companies and countries, are centered in efficiently managing knowledge, which is the intangible capital, the engine of added value, and the driver of economies and sustainable development.

\section{Introducción}

Entender los hitos más importantes de la relación ciencia y tecnología con el posgrado, implica darle relevancia a la formación de capital humano. Estos son el punto de partida del crecimiento y la equidad, dentro de una clara concepción de que con una formación de alto nivel y calidad se logra producir y socializar el conocimiento para un desarrollo sostenible en el largo plazo.

Las fuerzas productivas apoyadas en una infraestructura institucional académica, científica, de redes de información y con una vinculación activa con la sociedad, constituyen lo que se ha denominado el "capital social de la investigación" y del desarrollo científico y tecnológico. Es significativo, el interés de conocer cómo desde la universidad se genera valor al cono- cimiento; su gestión es una forma de responder a problemáticas del entorno apoyado en la contribución de personas e instituciones que durante décadas han ido transformando esa relación de investigación con la formación posgradual. Una aproximación que se plantea para la comprensión entre los primeros aportes de la investigación y posgrado, fue la trayectoria del marco histórico que dejaba de manifiesto el inicio de las primeras universidades a principios del siglo XII en donde la mayoría de las existentes surgían en el seno de la Iglesia y su misión fundamental era la de preservar el conocimiento y transmitirlo, principalmente a través de la moralidad, misticismo y religiosidad como sus rasgos más esenciales.

Ya durante los siglos XVI y XVII, las universidades entraban en una etapa de declive, a raíz de su tendencia conservadora, lo que favorecía la aparición de nuevas instituciones: las socieda-

*Autor de correspondencia.

E-mail:rahd17@gmail.com (Román Antonio Hernández Dávila)

(c) (i) () $(-)$ Peer review is the responsibility of the Universidad Francisco de Paula Santander.

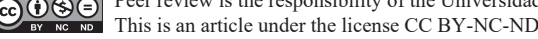


des científicas y academias, que se convertían en las protagonistas en el desarrollo de la investigación científica, de acuerdo con las necesidades de una sociedad cada vez más tecnificada $\mathrm{y}$ dando pie a un resurgir de la universidad en el siglo XIX.

A principios de ese siglo, Humboldt proponía un nuevo modelo de universidad que combinaría la función tradicional, la docencia, con una segunda función, la investigación, organizada mediante disciplinas especializadas, para su propio bien. A partir de ese momento la misión fundamental de la universidad era la de desarrollar el conocimiento científico puro con base empírica, y transmitirlo mediante la formación, la publicación y divulgación. Ya durante el siglo XIX y XX se reconocía la importancia del conocimiento como factor de producción, y la creación de valor en la sociedad contemporánea condiciona a las universidades para que a través de la investigación y la creación de nuevos conocimientos, sean los llamados a jugar un papel importante en el desarrollo de la región y en la innovación tecnológica y social.

Desde la perspectiva de Pavez [1], se considera que el rol del conocimiento en las universidades ha cambiado debido a los nuevos paradigmas inscritos a la sociedad de la información y la nueva economía basada en el conocimiento. Esta relación investigación -empresa encuentra en la innovación, un surgimiento en la tecnología.

El nuevo modelo de universidad está fuertemente influido por una aproximación al funcionamiento de una economía de mercado que favorece el desarrollo económico dando pie a uno de los grandes desafíos como es el de equilibrar de forma dinámica las tres misiones que actualmente tienen encomendadas: enseñanza, investigación y extensión.

Por otro lado, en aras de entender esta transitoriedad y considerando el avance científico y transcendental de la gestión del conocimiento, es pertinente mencionar que quienes planifican la formación universitaria de nuestros países en "vías de desarrollo", deben tomar como coordenada ineludible, que lo único permanente en esta "sociedad del conocimiento" es el cambio, lo cual conlleva a pensar en qué hacer en áreas vitales para el desarrollo, social, científico y tecnológico -que lo produce- como son la investigación y los postgrados, máxime cuando se tenga como criterio una conexión indisoluble entre estos componentes académicos.

La amplia brecha existente entre el mundo rápido y el lento se empezará a reducir, sí y solo sí, se logra aumentar ostensiblemente inversiones e innovaciones convertidas en bienes y servicios cuyo valor agregado a la economía y al intelecto social mejoren la calidad de vida y movilice la economía hacia niveles más productivos y competitivos. Sostiene Oppenheimer [2], al comparar el crecimiento económico de algunos países desarrollados con los nuestros, que el $40 \%$ del crecimiento de aquellos, proviene de hallazgos científicos y patentes incorporadas al mercado.Para llegar a esos niveles las instituciones públicas y privadas deben aumentar la inversión en investigación y desarrollo, así como solicitar resultados tangibles.

Entre los principales "problemas nodales", citados en el documento "Acuerdo por lo Superior 2034", [3] del CESU, aparece el que "la escasa inversión en ciencia, tecnología e innovación que representa solo el 0,46\% del PIB, muestra que el desarrollo científico no es prioridad real para el país" (N², 102). Más adelante se expresa "este $0,46 \%$ corresponde a un 0,19 asignado a COLCIENCIAS; el porcentaje restante está representado en los recursos procedentes de recursos propios de la IES y fuentes externas nacionales e internacionales" [3].

Como se observa, esta escasa inversión está muy por debajo de estándares internacionales que la ubican entre el 3 y 4 por ciento del PIB, para obtener resultados como los citados por Oppenheimer. Profundiza el problema, la escasa producción observada en otros indicadores de ciencia, tecnología e investigación, tales como “...la baja cifra de patentes, el escaso número de doctores y la escasez de publicaciones en revistas indexadas y en otros productos resultados de investigación" $[3]$.

Datos de COLCIENCIAS aparecidos en noticias UN indican que en Colombia por millón de habitantes, el país tiene 154 investigadores, mientras que la media en latinoamérica 538 y en el mundo es de 1285 (noticias UN- 23,12,2016). Se aduce la deficiente financiación del sistema de CTI, como causa principal de este problema y la inexistencia de un equilibrio en los recursos destinados a proyectos y programas doctorales [4].

Hay 6,6 profesionales con título de doctorado por cada millón de habitantes, un porcentaje bastante bajo si se tiene en cuenta que el promedio por igual proporción de personas en América Latina es de 38; lo cual permite concluir que si no se mejoran estas cifras se hace difícil hacer investigación de calidad.

Las cifras y datos enunciadas dan sustento al planteamiento de este trabajo respecto de la necesitad de repotenciar la colaboración mediante esfuerzos de convenir alianzas estratégicas que potencien creación de doctorados, redes telemáticas, y condiciones para vincular lo máximo posible la investigación con los postgrados [4].

Pese a esos indicadores hay expectativas positivas. El documento de COLCIENCIAS [3] sostiene que el conocimiento avanzado debe alcanzar al mayor número posible de personas, que por esa vía pueda "adaptarse en forma rápida y eficaz a los cambios". Todo el contexto general de ese trabajo insiste en crear "una estrecha relación entre investigación básica, investigación aplicada y el desarrollo" [4].

Se concluye que emprendimiento e innovación van de la mano cuando se dinamizan las visiones sobre las oportunidades que puedan aprovecharse para diversificar la economía, potenciar 
la productividad, y lograr desarrollos sustentables, mediante estrategias que propendan al "....aprovechamiento de recursos destinado al fomento de actividades CTel; para hacer visibles redes de cooperación y diálogo entre los actores involucrados en la CTel nacional, y para establecer cuáles son las áreas prioritarias de acción,conllevando a implementación de políticas de fomento e inversión en el sector." [4].

La investigación está íntimamente relacionada con la innovación y el desarrollo de las fuerzas generadoras de producción y riqueza, material, intelectual y social. Piketty sostiene que llegar a las etapas de desarrollo planteadas por el PNUD, vinculadas no sólo al ámbito económico, sino también de educación, salud y derechos fundamentales, requieren apoyar con denuedo condiciones institucionales propicias a obtener nuevos conocimientos en éstas áreas, que, de concretarse, supondrán logros en equidad, sostenibilidad, cohesión social y mayores oportunidades para obtener un nivel de vida decente [5].

Los diagnósticos hechos por organismos internacionales, tales como: la UNESCO, el PNUD, la CEPAL, entre otros, ocupados en analizar los aspectos claves del mejoramiento de la calidad en las universidades, concluyen que éstas ya no monopolizan la producción del conocimiento avanzado y deben coincidir con otros organismos e instituciones públicas y privadas, nacionales e internacionales, para aplicar nuevos formatos en la creación de conocimientos que incluyan alianzas estratégicas tendentes a vinculaciones inter disciplinares y procesos de innovación productiva y tecnológica (Fig. 1).

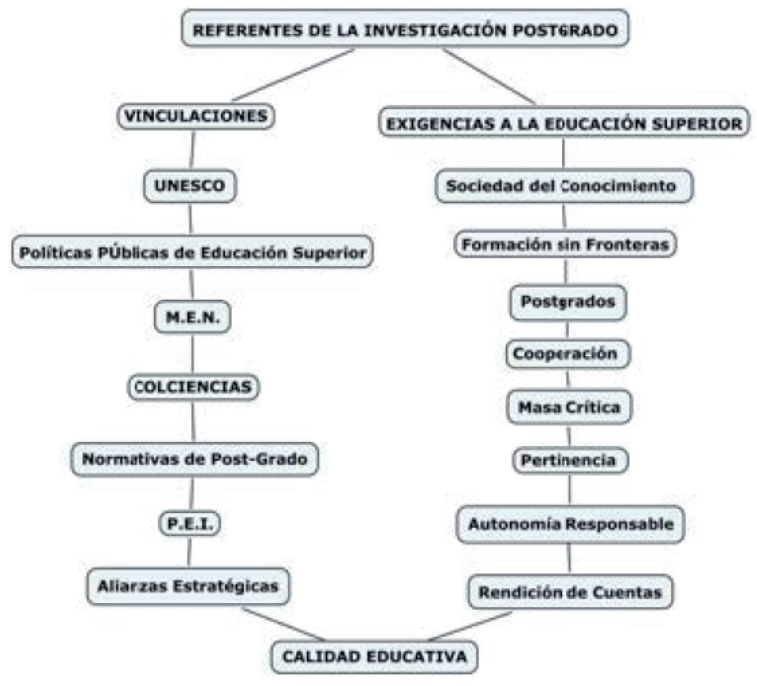

Figura 1. Referentes de las investigación postgrado

Los logros académicos de las IES (Institución de Educación Superior), deben reflejarse en la formación de personas capaces de adaptarse a las transformaciones de los entornos cercanos y mediatos, e insertarse como sujetos activos y productivos en la sociedad. Esas metas las potencia el acoplamiento entre producción científica (investigación) y postgrado, y refleja un síntoma de calidad en las instituciones que las asumen, lo cual es factor fundamental para impulsar la demanda hacia ellas.

Además de los esfuerzos endógenos para mejorar la relación investigación-postgrados, se hace necesario agregarles valor con iniciativas generadoras de diálogo de saberes en aras de confluir en la construcción del pensamiento transformador.

Las alianzas con grupos de investigación de otras universidades, la detección de nuevas líneas de investigación que surjan del intercambio, así como del valioso capital intangible que los activos cognitivos interinstitucionales produzcan, son garantía de mejorar las ofertas de postgrado y de investigar para resolver necesidades y problemas planteados en esos escenarios integrados.

Este planteamiento implica mejorar la productividad científica con base en la cooperación entre instituciones de educación superior. Así también lo vislumbra la UNESCO en el documento: Situación Educativa de América Latina y el Caribe [6], al recomendar "generar una capacidad propia de producción científica y tecnológica...aumentar la inscripción de patentes...contar con una masa crítica de científicos, y de jóvenes formados a nivel de doctorado en programas de nivel internacional...es un desafío que, por su escala, no se podrá encarar de manera aislada, por lo que se debe intensificar la cooperación.” [6].

La investigación concebida con esos parámetros, concuerda con planteamientos como los surgidos en la "Declaración de la Conferencia Regional de Educación Superior en América Latina y el Caribe-CRES 2008", de Cartagena, donde taxativamente se planteó que las universidades "...deben inducir el desarrollo de alternativas e innovaciones en las propuestas educativas, en la producción y transferencia de conocimientos y aprendizajes, así como promover el establecimiento y consolidación de alianzas estratégicas entre gobierno, sector productivo, organizaciones de la sociedad civil e instituciones de educación superior, ciencia y tecnología." [6].

\section{La región, las IES y sus reflexiones instituciona- les}

Las IES tienen amplios referentes en sus documentos vitales, tales como los proyectos institucionales, sobre aportes al desarrollo regional; en los cuales incorporan en su misión y visión, planteamientos sobre el desarrollo de la investigación imbricados en resolver problemas regionales, nacionales y del mundo, necesarios de visibilizar en resultados tangibles, que apoyen el reconocimiento académico otorgado por las instituciones cualificadas para ello. También incorporan como soporte de su vida académica, la investigación con énfasis en la innovación y la tecnología, dirigidas a solucionar problemas de esa naturaleza. Si la brújula que oriente el destino de la investi- 
gación tiene coordenadas en las necesidades regionales y estas pudieran explorarse con apoyo de instituciones económicas, sociales, gubernamentales, entre otros, que forman parte del entorno, estaríamos hablando entonces, de buscar nuevos conocimientos con pertinencia en el desarrollo sostenible; eslabón necesario en la cadena del progreso socioeconómico nacional.

Los Planes de Desarrollo Regionales y Nacionales enuncian políticas públicas sobre el mejoramiento de la calidad de vida, mediadas por acuerdos con instituciones de educación superior interesadas en asumirlas en cooperación, y soportadas en el uso de las regalías regionales creadas para financiar tales fines. Con base en ellas pueden concertarse interesantes convenios en áreas de: fronteras e integración, educación, tecnologías, desarrollo sustentable, reducción del desempleo, factibles de acordar para investigar. [7] Sin embargo, es importante acotar, respecto de las regalías, la necesidad de establecer mecanismos de control fiscal sobre ellas. Como se sabe el 10\% del monto total en regalías debía destinarse a proyectos del Fondo para Ciencia, Tecnología e Investigación. El periódico El Tiempo informa que en el año 2016, 1.5 billones de pesos correspondiente a ese renglón no fue ejecutado en las regiones [8]. Se habla de pretender reformar el sistema de regalías para invertir esos recursos en infraestructura terciaria, por tanto, por este año se perdería el destino original de esa inversión.

Si nuestras instituciones muestran resultados positivos en los aportes al desarrollo regional, se convertirían en vitrinas propicias a mayor interés en la oferta de sus carreras, y así obtener más recursos para sustentar proyectos factibles en investigación.

También se pudiera, con tino, lograr un paulatino acercamiento con sectores productivos para efectuar convenios en los que alumnos de postgrado se vinculen, vía trabajos de grado, a la resolución de problemas en los ámbitos de comercio, productividad, gestión gerencial, los sistemas administrativos, financieros, o de competencias informáticas y tomas de decisiones.

El Tecnológico de Monterrey, sobre este aspecto, incorpora el concepto: "Aprendizaje Basado en Retos" (ABR), en su modelo educativo TEC21, para acoplar sus principios a "experiencias abiertas de aprendizaje", mediante acuerdos, que permitan vivencias para exponer al alumno a situaciones desafiantes del entorno, cuyo sustento obvio es la investigación [9].

Los planteamientos sostenidos supra, llevan a propuestas como las de Asociación Colombiana de Universidades (ASCUN), de contribuir en experiencias proclives a la construcción del tejido social y empresarial. La academia, el sector productivo, el gobierno nacional y los gobiernos regionales son los actores principales en la dinámica de la relación Universidad Empresa-Estado, la cual se ha venido fortaleciendo cada vez más en las diferentes regiones del país. Esta relación significa para las regiones poder acercar a los docentes, investigadores e instituciones a las realidades de las necesidades de cada sector productivo y encontrar, por medio de sus proyectos articulados de investigación, soluciones prácticas e innovadoras [10].

Existen experiencias en las que mediante convenios inter institucionales universitarios, se atiende desde los postgrados como lo plantea COLCIENCIAS, necesidades de los sectores productivos con trabajos de grado de investigación aplicada, cuyos objetos de estudio son soluciones a problemas identificados en empresas, o visualizadas desde allí. [3] Nuestras instituciones, tienen potencial y experiencia para sostener servicios de esa índole, que por efecto colateral positivo, fortalecería el crear nuevas líneas y grupos de investigación, e interés académico para impulsarlas. Necesario es mencionar que, el concepto de desarrollo regional está asociado a las dinámicas sociales que identifican a amplios conglomerados humanos, con historias y culturas diversas, pero comunes, ubicados en determinados espacios geográficos. El focus de atención de la investigación científica en la universidad, debemos dirigirlo también hacia estas dinámicas con criterios interdisciplinario, cualitativo, etnográfico y heurístico, de impacto trascendente. Lo que los grupos humanos intuyen como suyo en el imaginario colectivo, es digno de estudiarse y difundirse desde estrategias investigativas de pertinencia social y cultural. Estos aspectos deben verse también asociados a los objetivos de los semilleros, y así vislumbrar problemas de esta esfera epistémica, asociados a los intereses y expectativas de las comunidades donde habitan sus alumnos integrantes. Indagar los problemas en los contextos de origen forma parte de los compromisos prioritarios de la educación superior, según el ya citado documento de Cartagena, y crea espacios para las agendas de investigación en defensa de nuestros patrimonios culturales y respeto a nuestras identidades e idiosincrasia.

\section{La formación por competencias, la innovación y el pregrado}

El logro de competencias (saber, saber hacer, saber actuar, saber ser), cresta de la ola contemporánea en la formación universitaria, para el mejor desempeño profesional enfoca la investigación como eje transversal. En correspondencia con ese criterio formativo, se parte de la base de que los resultados de los trabajos de grado deben corresponder a los siguientes objetivos:

a) Identificar aquellos de mayor calidad y pertinencia en las titulaciones ofrecidas por la institución, a objeto de que sean referencias actualizadas a los propósitos docentes de la academia y puedan servir a la creación de nuevas líneas de investigación [11]; en tanto que éstas deben ser definidas teniendo en cuenta prioridades de los grupos de investigación y en corres- 
pondencia con las necesidades del entorno.

b) Premiar a sus creadores para que faciliten esas experiencias, de resultados aplicados, exponiéndolas como invitados en las cátedras afines a las áreas de conocimiento pertinentes.

c) Operar con grupos de trabajo multidisciplinarios Universidad-Empresa-Estado comprometidos y disponer de un articulador confiable, con recursos que alcancen un alto nivel de financiamiento múltiple de apoyo a los grupos que logren mejores expectativas acordes a los intereses en investigación.

En las perspectivas de la investigación dentro de la misión y visión universitarias, está formar talento y noveles investigadores, por lo que sería de interés para nuestras instituciones de educación superior, conocer directamente de sus autores vivencias de ésta naturaleza y productos de innovaciones, conectados con contextos internos y externos que fomente la internacionalización del conocimiento. Como bien lo afirma Chesbrough [12] "No se puede cumplir con los objetivos de crecimiento si se ignora la gran cantidad de gente brillante que está ahí afuera y no se encuentra en su nómina”.

\section{¿Innovar para qué?}

Innovar implica, incorporar la idea de cambio en los contextos de análisis y objetos de estudio. Investigar pues, para resolver problemas fácticos y que la acción reflexiva proponga soluciones que demuestren su eficiencia respecto de la situación inicial tomada como necesidad a resolver, para estimular la búsqueda interdisciplinaria en la construcción del conocimiento como elemento cotidiano determinante de la formación profesional continua de los docentes a partir de su propia práctica educativa.

Las características que deben establecerse en los trabajos de investigación y la estricta evaluación que se haga de ellos, demostrarían su acople a lo que es innovar; en el sentido, de que la acción reflexiva humana puede avisorar problemas o necesidades, grandes o pequeños, localizados en ámbitos específicos para que mediante intervenciones planificadas se presente el "deber ser" que los solucione.

Es necesario crear escenarios en los cuales los autores muestren sus novedosas experiencias o productos investigativos, en la idea de propiciar sistemas relacionales comunicativos, mediante la reflexión - acción que permitan plantear interrogantes y responder acerca de los modos diversos de llegar a los cambios innovadores que conllevan a pensar lo posible para hacerlo realizable. Tanto las líneas de investigación como los semilleros y aquellas cátedras que estimulan la adquisición de la competencia investigativa, se beneficiarían de estas iniciativas, las cuales pueden presentarse a instituciones públicas y/o privadas interesadas en acuerdos estratégicos.
De iniciativas como las planteadas, pueden surgir ideas de emprendimiento empresarial incipientes como las contempladas en la aplicación del concepto en inglés "star up", propicias para el desarrollo tecnológico relacionado con el mundo del internet y las tics, o, innovar en áreas donde las IES puedan aportar iniciativas productivas o del área social. Otro ejemplo, puede ser las denominadas "spin off", cuyas raíces son empresas ya existentes, donde las IES pudieran, gracias a sus investigaciones, aportar ideas para la diversificación productiva o creación de una nueva. Es emblemático como ejemplo, el surgimiento de lenovo, que es una spin off de IBM.

La innovación abierta es fundamentalmente una nueva forma de lograr cambios para el desarrollo sostenible [12]. La idea principal es que, en la actualidad, el conocimiento está diseminado, de forma que ninguna empresa o institución, en este caso la universidad, tiene el monopolio de las grandes ideas. Pero se requiere del pensamiento complejo para conseguir relaciones sistémicas entre partes epistémicas que deben colaborar en fines comunes.

Con la propuesta de aplicación de éste modelo se requiere que las investigaciones lleven asociadas para la mejora del conocimiento en las universidades, vinculaciones estratégicas con agentes e instituciones que vislumbren objetos de estudio, superen necesidades regionales o nacionales y propongan modelos de desarrollo sostenible con pertinencia social, económica, científica y humanística.

En síntesis, el autor mencionado relaciona la innovación con esfuerzos individuales y de grupo; iniciativas disciplinares de relación educación-tecnología; exigencias del currículo; iniciativas institucionales sistémicas, y sus derivados que emergen de las concepciones pedagógicas y exigencias de nuevas normativas que se puedan crear.

\section{Redes académicas y su utilidad}

La idea de la formación sin fronteras y el uso de las TIC's se ha incorporado con potencia cada vez mayor para fomentar el intercambio académico que en materia de investigación y postgrado es un recurso fuente del diálogo de saberes necesarios en la construcción del pensamiento transformador, y es eje de los aportes que las instituciones autónomas deben hacer al país en las áreas científica y humanista.

\section{Metas de intercambio académico}

¿Cuáles metas pueden potenciar las políticas de intercambio basadas en la creación de redes académicas?:

a) Revisar posibilidades de alianzas estratégicas con otras 
instituciones nacionales e internacionales, a objeto de crear postgrados que acrediten la producción de conocimientos; para lo cual, se establecerán condiciones de interdependencia con autonomía. Ellas deben firmarse posterior a desarrollar una secuencia de aproximaciones que abra espacios para reducir la resistencia y muestre las bondades que la integración genera.

b) Evaluación de posibilidades de acuerdos interinstitucionales, tendientes a crear postgrados itinerantes, presenciales, semi-presenciales o a distancia.

c) Gestionar redes telemáticas respecto de objetos de estudio compartidos con otras instituciones universitarias y/o privadas, públicas u organizaciones no gubernamentales.

d) Propiciar a través de las redes, comunidades de intercambio (Webinar) cuyo propósito sea el trabajo colaborativo interdisciplinario y transdisciplinario, sobre temas de relevancia fundados en prioridades nacionales e internacionales.

e) Crear una página web y blog donde se expongan opiniones sobre los temas de discusión adelantado por los investigadores; en ella se abrirá un foro virtual que debata sobre las propuestas de investigación o de postgrado que surjan.

f) Determinar con base en diagnósticos y estudios de factibilidad en conjunto, qué tipos de postgrados se pueden ofrecer cuando se trate de creación.

g) Informar sobre fuentes de financiamiento externo e interno que alimenten los postgrados para su funcionamiento.

h) Crear una base de datos donde aparezcan:

Planificación de las aproximaciones que abra espacio para los acuerdos;

El tiempo disponible que puedan utilizar los profesores;

Escalafón,experiencia y títulos que poseen los profesores;

Información sobre equipamiento y estructura física, laboratorios, en especial de teleinformática que tengan cada institución integrante del acuerdo;

líneas y proyectos de investigación existentes;

Grupos y núcleos de investigación activos;

Potenciales objetos de estudio y fortalezas que tienen las instituciones.

\section{Requisitos de los postgrados}

Respecto de la posibilidad de crear postgrados con base en propuestas surgidas de la creación de redes, estas deben sustentarse en los siguientes requisitos:

a) Es indispensable que el programa académico sea acreditado en el país de origen por el organismo encargado para tal fin.

b) El programa debe tener correspondencia con la oferta del pregrado de la universidad receptora para que sirva a su mejora. No hay investigación de calidad si no contribuye a mejorar la calidad de la docencia (Tummerman: 1996). Innovar, propiciar mejores prácticas pedagógicas, y reajustar el currículo son objetivos indispensables en estos acuerdos.

c) Contar con personal calificado para la gestión del convenio y exigir a la unidad académica de adscripción en la universidad receptora, una adecuada infraestructura física, orgánica y de equipamiento.

d) Que estimule el intercambio, la cooperación, el surgimiento de otras experiencias, tales como: actividades de extensión, de capacitación, conferencias, congresos, líneas de investigación, etc.

e) Exigir expresamente la participación de personal docente de la universidad receptora en el desarrollo de las experiencias.

f) La universidad que imparta el postgrado debe ser de calidad, presentar un plan de estudio aprobado con éxito y contar con excelentes profesores.

g) Se debe exigir la contextualización del plan de estudios a las características de nuestras necesidades sociales y preocupaciones académicas, previas negociaciones entre los coordinadores de gestión (de las universidades integrantes del convenio) y apunte a implementar las tendencias curriculares contemporáneas respecto a que sea abierto, flexible, y otorgue la posibilidad al participante de construir su propio currículo de acuerdo a su tiempo e intereses.

h) Que ayuden a fomentar sistemas integrados de información académica, nacional e internacional, publicaciones periódicas.

i) Que sean económicamente factibles y se apoyen en diversas fuentes de financiamiento: COLCIENCIAS, gobernación, UNESCO, PENUD, empresas privadas.

j) Que sean destinadas a formar la generación de relevo en la institución receptora, así como crear condiciones para que, de cara al futuro, potencie la creación de postgrados propios, en particular de doctorado.

k) Monitoreo de la calidad del programa, por pares con experiencia en evaluación institucional, de las instituciones involucradas, para valorar su eficiencia respecto de los términos que éste contiene y de los beneficios que pueda desplegar 
hacia la mejora del sistema nacional de CTel.

\section{Ventajas de los postgrados interinstitucionales}

Grosso modo, la implantación de postgrados con estas características presenta las siguientes ventajas:

a) Contribuye a la formación de una masa crítica con impacto en la disminución del déficit de investigadores, y en la conformación de un tejido investigador, necesario para fortalecer el sistema CTel, además de potenciar la presencia con fortaleza de la creación científica nacional en los escenarios donde se debaten las ideas de cambio.

b) Las visiones integradas, forjadas en el intercambio, ayudan a florecer objetos de estudio y contenidos temáticos del contexto nacional, responsivo a la solución de problemas y necesidades propias que evitan el desarraigo de futuros investigadores.

c) Sustenta la vinculación entre pares científicos que ayuda a crear grupos y líneas de investigación para el trabajo científico en colaboración.

d) Baja los costos, porque se comparten, y enriquece los beneficios académicos, merced al intercambio, la socialización del conocimiento, el aumento de innovaciones, el beneficio del pregrado, el incremento de tutores y la formación de la generación de relevo.

e) Propician las comunidades de aprendizaje (Learning communities) al incentivar que los profesores exploren enfoques, metodologías y herramientas y desarrollen competencias para la formulación e impulso de iniciativas en el fortalecimiento de sus prácticas pedagógicas.

f) Fortalecen las competencias, tanto generales como específicas y la asunción de los roles profesionales, si se planifican para favorecer la integración de saberes que conlleven a elaborar proyectos finales propiciadores de soluciones a problemas particulares con criterios interdisciplinarios asumidos en sus períodos académicos (Fig .2).

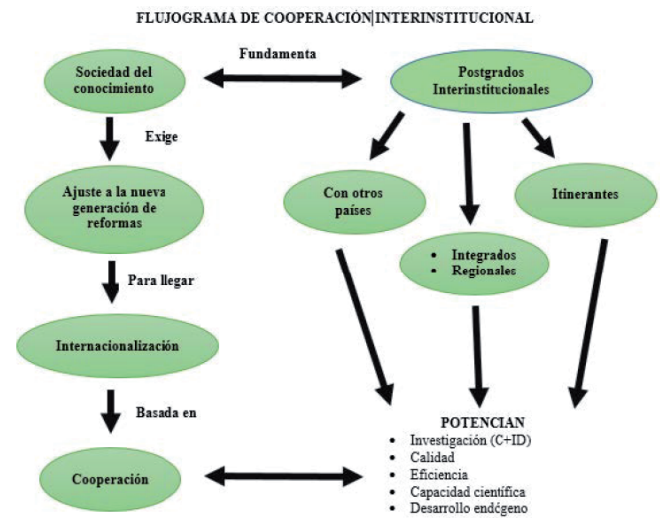

Figura 2. Flujograma de cooperación interinstitucional

\section{Conclusiones}

Los planteamientos recogidos en las páginas precedentes, ponen de manifiesto que la cooperación y la investigación con criterios de pertinencia juegan un papel importante para afrontar con propiedad los tres desafíos que Morín [13] confiere al conocimiento para superar su fragmentación: a) mayor comprensión de lo global; b) de lo complejo y sus relaciones; c) de la expansión descontrolada y veloz del conocimiento.

En esa dirección, es decisiva la complementación investigación -posgrado para que las instituciones universitarias aporten a los desarrollos sustentables que requieren nuestros países e incorporen la excelencia científica en ese campo. Lograr esos niveles de mejora contribuirá a crear progreso en los objetivos de la investigación en cuanto al capital social e intelectual y el desarrollo (C+ID) de forma conjunta [14].

Pensar en una relación investigación-postgrado, supondrá abordar también la gestión de la universidad para que ella se encargue de ampliar las oportunidades educativas vinculadas a la producción de conocimiento,mejorar los perfiles profesionales, crear innovaciones y lograr competencias útiles en el desempeño profesional.

\section{Referencias}

[1] A. Pavés, Modelo de implantación de Gestión del Conocimiento y Tecnologías de Información para la Generación de Ventajas Competitivas. Valparaíso, Chile: Universidad Técnica Federico Santa María, 2012.

[2] A. Oppennheimer, Crear o morir. Bogotá, Colombia: Debate, 2015.

[3] Consejo Nacional de Educación Superior, Acuerdo por lo superior 2034. Bogotá, Colombia: CESU, 2014.

[4] Departamento Administrativo de Ciencias, Tecnología e Innovación - Colciencias, Modelo de medición de grupos de investigación, desarrollo tecnológico o innovación y de reconocimiento de investigadores del sistema nacional de ciencia, tecnología e innovación. Bogotá, Colombia: Colciencias, 2015. [5] T Piketty, El capital en el siglo XXI. Paris, Francia: Éditions du Seuil, 2013.

[5] Organización de las Naciones Unidas para la Educación, la Ciencia y la Cultura, Situación Educativa de América Latina y el Caribe. Santiago, Chile: Imbunche, 2013.

[6] Organización de las Naciones Unidas para la Educación, la Ciencia y la Cultura, Declaración de la Conferencia Regional de la Educación Superior en América Latina y el Caribe. 
Santiago, Chile: UNESCO, 2008.

[7] Departamento Nacional de Planeación, Bases del Plan Nacional de Desarrollo. Bogotá, Colombia: DNP, 2014.

[8] Diario El Tiempo, "Sacar la política de la ciencia," [En Línea]. Disponible: http://www.eltiempo.com/opinion/editorial/sacar-la-politica-de-la-ciencia-24-de-marzo-de-2017-708 54. Acceso Abr. 24, 2017.

[9] Tecnológico de Monterrey, "Modelo Educativo del Tecnológico de Monterrey". [En Línea]. Disponible: http://www.itesm.mx/wps/wcm/connect/ITESM/Tecnologico+de+Monterrey/ Nosotros/Modelo+educativo/

[10] Ascun , Asociación Colombiana de Universidades , Acuerdo $\mathrm{N}^{\circ} 01$ de Junio 9 de 2006. Consejo Nacional de Rectores, 2006

[11] V. Kumar, "A process for practicing design innovation," J. Bussines strategy, vol. 30, no. 2/3, pp.91- 100, Feb. 2009

[12] H. Chesbrough, Innovación de servicios abiertos: reinvente su negocio para crecer y competir en una nueva era. Madrid, España: Plataforma, 2011

[13] E. Morin, La cabeza bien puesta. Repensar la Reforma. Reformar el pensamiento. Buenos Aires, Argentina: Nueva Visión, 2001.

[14] E. Bueno, "El capital social en el nuevo enfoque del capital intelectual de las organizaciones," Psicol. Trab. y Org, vol. 18, no.2/3, pp. 157-176, Mar. 2002 